\title{
The Effects of Nigella Sativa on the Immune Disorders
}

\author{
AFTAB HOSSAIN ${ }^{1}$ \\ ${ }^{1}$ University of Calcutta
}

May 6, 2020

\begin{abstract}
Numerous studies have been carried out over the years to substantiate the claims that Nigella sativa oil or Kalonji oil has immune-modulatory properties and has a positive effect on diverse conditions resulting from deficiencies or abnormalities of the immune system. In this review article, few clinical trials have been looked into in order to determine the specific effects of NS on the immune system and whether the existing literature is substantive.
\end{abstract}

Kalonji, Sehat Amrit, Naturoveda

\section{Introduction}

Nigella sativa is an annual herb with several medicinal properties. Literature relating to the prescription of Nigella sativa in various diseases go back 2000 years in history. Black seed oil has been commonly used in the treatment of health issues relating to the cardiovascular system, liver \& kidney functions, digestive tract, respiratory system and immune system support, besides overall well-being. The rich folklore surrounding Nigella sativaoil, especially in the Indian and Arabic traditional medicinal literature is the inspiration behind most of the clinical trials that have been carried out to determine its specific pharmacological benefits as well as the dosage in which it should be consumed to derive those benefits without any side effect. Thymoquinone is the prime among the numerous active components present in Kalonji like fatty acids, flavonoids, saponins, alkaloids, etc. Most of the medicinal properties of $N S$ owe their origin to thymoquinone.

\section{Materials \& Methods}

Secondary research has been used extensively to understand the immuno-modulatory properties of Nigella sativa. To that effect, authentic clinical trials that showed the direct effect of Kalonji on the immune system have been studied. As Immune System modulation is a significant trait of Nigella Sativa, there is no dearth of research literature pertaining to it. In addition to that, other studies in respect of allergy-related conditions like allergic rhinitis, atopic eczema and asthma have also been consulted.

\section{Results \& Discussion}

\section{In autoimmune disorder Vitiligo}

Vitiligo is an auto-immune disease that affects the skin. It causes discolouration of skin in blotches. In some cases, it also affects hair and inside of the mouth. In a study to compare the effect of Nigella sativa oil and fish oil on vitiligo lesions, randomized sampling was done on 52 patients. They were divided into 2 equal groups and the medications were applied by one group twice a day on the vitiligo lesions. This double-blind trial was carried out between June \& December 2011 by the dermatology clinic of the Imam Khomeini Hospital Ahvaz, Iran. The rate of improvement of lesions was accessed by the Vitiligo Area Scoring Index (VASI) after 6 months. A P value $<0.05$ was considered statistically significant after the collected data was analyzed using SPSS v. 15. 
The VASI mean score decreased from 4.98 to 3.75 in patients who appliedNigella sativa oil for 6 months, while for those applying topical fish oil went down from 4.98 to 4.62 only. Major improvements were observed

in the neck, head, trunk and upper extremities of those who used Nigella sativa, and most of the improvement was centred on the feet, trunk, neck \& head for those who received fish oil1.

1 Ghorbanibirgani et al , 2014. Comparing Nigella sativa oil and fish oil in treatment of vitiligo .

2 Ahmed et al , 2014. Evaluation of efficacy, safety and antioxidant effect of Nigella sativa in patients with psoriasis: A randomized clinical trial.

3 Nikakhlagh et al , 2011. Herbal treatment of allergic rhinitis: the use of Nigella sativa .

4 Ansari, 2010. Montelukast versus nigella sativa for management of seasonal allergic rhinitis: A single blind comparative clinical trial .

5 Alsamarai et al, 2014. Evaluation of topical black seed oil in the treatment of allergic rhinitis.

6 Işlk et al, 2010. Potential adjuvant effects of Nigella sativa seeds to improve specific immunotherapy in allergic rhinitis patients.

7 Dirjomuljono et al ,2008. Symptomatic treatment of acute tonsillo-pharyngitis patients with a combination of Nigella sativa and Phyllanthus niruri extract.

\section{In skin disorder Psoriasis}

Psoriasis is a hyperproliferative skin condition which can be itchy and is sometimes quite painful. It is autoimmune in nature. To compare the antipsoriatic effect of the ethanolic extract of Nigella sativaand asiaticoside, a comprehensive experimental study was carried out by Ahmed et al. In the study, Nigella sativa was administered in 2 forms, as an oral dosage and as an ointment. They had IC50 value of 23.9 $\mu \mathrm{g} / \mathrm{ml}$, which is about the IC50 value for asiaticoside $(20.13 \mu \mathrm{g} / \mathrm{ml})$. It was observed that Nigella sativa has a better antipsoriatic effect than the treatment in comparison. It concluded that the benefits of Nigella sativa can be best derived for psoriasis when it is applied in both ointment and dosage forms in combination2.

\section{In inflammatory condition Allergic Rhinitis}

Characterized by itching, nasal congestion, rhinorrhea \& sneezing, allergic rhinitis is a kind of inflammatory response of the nasal mucosa to natural allergens3. When comparing the therapeutic effects of montelukast (10mg/day) and that of Nigella sativa seeds $(250 \mathrm{mg} /$ day) on patients with seasonal allergic rhinitis over 2 weeks, it was seen that both resulted in visible improvement in ophthalmic \& daytime symptoms. However, Nigella sativa was more efficient in alleviating the nighttime symptoms4.

In another study conducted by Alsamarai et al , it was demonstrated that nasal drops of Kalonji oil considerably improved the symptoms in allergic rhinitis patients as well as their ability to withstand exposure to allergens5. The study included the application of 2 drops of Nigella sativa oil (one in each nostril) thrice daily, for 6 weeks. Each drop comprised $15 \mathrm{ml}$ of oil.

A separate study showed that allergic rhinitis patients undergoing allergen-specific immunotherapy for a month and then taking Kalonji seeds (2g/day) along with immunotherapy the next month experienced better progress (PMN functions \& CD8 counts) than those that just underwent immunotherapy for 2 months6. It concluded that administration of Nigella sativa contributed towards more effective immunotherapy.

\section{In patients with Tonsillopharyngitis}

Research undertaken by Dirjomuljono et al showed that NSPN capsules comprising Nigella sativa (360 mg) \& Phyllanthus niuri $(50 \mathrm{mg})$ could considerably reduce the disease's symptoms. These benefits are accredited to the immune-modulatory \& anti-inflammatory effects of Nigella sativa7 . 
In another study, it was found that the percentage of lymphocytes \& the NK cell count considerably improved in patients diagnosed with inhalation allergy but to the positive effect of Nigella sativaon the patients' immune system8.

8 Deurer et al, 2002. Effect of black cumin oil on the immune status of patients with inhalation allergy.

9 Gheita and Kenawy, 2012. Effectiveness of Nigella sativa oil in the management of rheumatoid arthritis patients: a placebo controlled study. 10 Osman et al , 2012. Immunomodulatory effect of Nigella sativa oil treatment in iron deficiency anemia caused by refractory coeliac disease.

\section{In patients suffering from Rheumatoid Arthritis}

Atrophy of bones, degeneration of collagen fibres in mesenchymal tissues \& inflammation of joints are the common characteristics of chronic Rheumatoid Arthritis. It is true that the exact cause of the condition is not well-substantiated. However, a major role of autoimmunity has been indicated in the disease. The symptoms \& activity score of RA significantly improved in 40 patients who were administered placebo in the first month followed by Nigella sativa oil capsules $(500 \mathrm{mg})$ the next month in a particular study9. The modulatory effect of Nigella sativa on the immune system could be credited for these benefits.

\section{In Celiac Disease}

The symptoms of Celiac Disease are increased gluten sensitivity and inflammation \& destruction of the mucosa layer of the small intestine due to an autoimmune activity. Consumption of Nigella sativa oil capsule $(450 \mathrm{mg}) 2$ times a day along with a gluten-free diet for a period of 12 months was more effective than just a gluten-free diet in treating patients with iron deficiency anaemia caused by refractory Celiac disease in a research carried out by Osman et al . Visible improvement in the duodenal histology recovery, as well as immunological \& haematological indices, were observed10.

Characterized by chronic dermatitis, a burning sensation \& itchiness Dermatitis herpetiformis (DH) is an autoimmune disease caused by Celiac Disease. It was found that adding Nigella sativa oil capsules to a gluten-free diet enhanced the efficiency of the diet itself in the treatment of the condition in a separate study.

\section{Independent Research}

Looking at the numerous research that indicates the immune-balancing action of Nigella sativa, the R\&D department at Naturoveda Health World undertook an independent study. They administered different doses of virgin Nigella sativa oil to the patients afflicted with diverse chronic conditions and autoimmune disorders. In addition to that, they also prescribed different concentrations of this oil to the patients in order to determine the one with maximum efficacy.

After a long drawn out survey, it was found that Nigella sativa oil was effective in modulating the immune system to keep it at its peak, thus improving overall health and disease-resistance. 50 patients suffering from Rheumatoid Arthritis were administered $\frac{1}{2}$ tsp $(2.5 \mathrm{ml})$ Nigella sativa oil for 3 weeks. The participants not only experienced a reduction in pain but the stiffness also came down to varying degrees. On the other hand, 30 psoriasis-afflicted patients were given the same dosage $(2.5 \mathrm{ml})$ of Nigella sativa oil for 20 days. Their responses were sorted through a clinical software to generate a score between 1 to 10 to determine the efficacy of this oil. With 10 being the highest and 1 the lowest, 28 out of 30 participants had scores between 5-7 and 2 of them had scores of 3and 5 respectively. Virgin Nigella sativa oil also proved to be hugely beneficial in diseases like Allergic Rhinitis, Vitiligo and metabolic disorders. But more importantly, it rejuvenated the patients physiologically rather than just addressing the particular conditions. Almost all of the participants reported an increase in energy and vitality. And it had no side-effect.

They formulated an orally administered medicinal solution prepared with virgin Nigella sativa oil and named it Sehat Amrit, keeping in mind its numerous beneficial effects on the body.

\section{Conclusion}


It can be positively concluded that Thymoquinone, the active compound in black seeds have been shown to be beneficial to a significant extent for patients suffering from several auto-immune disorders. This may be due to the immuno-modulatory action of Nigella sativa.

\section{References}

Ahmed Jawad, H., Ibraheem Azhar, Y. and Al-Hamdi Khalil, I., 2014. Evaluation of efficacy, safety and antioxidant effect of Nigella sativa in patients with psoriasis: A randomized clinical trial. J Clin Exp Invest www. jceionline. org Vol, 5(2).

Ansari, M.A., Ansari, N.A. and Junejo, S.A., 2010. Montelukast versus nigella sativa for management of seasonal allergic rhinitis: A single blind comparative clinical trial. Pak J Med Sci April-June, 26(2), pp.249254.

Deurer, A., Schleicher, P., Kalus, U., Pruss, A. and Kiesewetter, H., 2002. Effect of black cumin oil on the immune status of patients with inhalation allergy. BIOLOGISCHE MEDIZIN, 31(2), pp.75-78.

Dirjomuljono, M., Kristyono, I., Tjandrawinata, R.R. and Nofiarny, D., 2008. Symptomatic treatment of acute tonsillo-pharyngitis patients with a combination of Nigella sativa and Phyllanthus niruri extract.International journal of clinical pharmacology and therapeutics ,46 (6), pp.295-306.

Gheita, T.A. and Kenawy, S.A., 2012. Effectiveness of Nigella sativa oil in the management of rheumatoid arthritis patients: a placebo controlled study. Phytotherapy research , 26 (8), pp.1246-1248.

Ghorbanibirgani, A., Khalili, A. and Rokhafrooz, D., 2014. Comparing Nigella sativa oil and fish oil in treatment of vitiligo. Iranian Red Crescent Medical Journal , 16 (6).

Işık, H., Çevikbaş, A., Gürer, Ü.S., Kıran, B., Üresin, Y., Rayaman, P., Rayaman, E., Gürbüz, B. and Büyüköztürk, S., 2010. Potential adjuvant effects of Nigella sativa seeds to improve specific immunotherapy in allergic rhinitis patients. Medical Principles and Practice, 19(3), pp.206-211.

Mohamed Alsamarai, A., Abdulsatar, M. and Hamed Ahmed Alobaidi, A., 2014. Evaluation of topical black seed oil in the treatment of allergic rhinitis. Anti-Inflammatory 83 Anti-Allergy Agents in Medicinal Chemistry (Formerly Current Medicinal Chemistry-Anti-Inflammatory and Anti-Allergy Agents), 13 (1), pp.75-82.

Nikakhlagh, S., Rahim, F., Aryani, F.H.N., Syahpoush, A., Brougerdnya, M.G. and Saki, N., 2011. Herbal treatment of allergic rhinitis: the use of Nigella sativa. American journal of otolaryngology ,32 (5), pp.402407.

Osman, M.T., Taha, B.I., Al-Duboni, G. and Muhamed, L.A., 2012. Immunomodulatory effect of Nigella sativa oil treatment in iron deficiency anemia caused by refractory coeliac disease. Res. J. Pharm. Biol. Chem. Sci, 3(4), pp.887-895. 